\title{
Internet Mentalization Informed Video Feedback (MI-VF) Intervention to Improve Parental Sensitivity in Mother-Infant Dyads with Maternal Depressive Symptoms: Study Protocol for a Randomized Controlled Feasibility Trial
}

\section{Marcia Olhaberry Huber}

Pontifical Catholic University of Chile: Pontificia Universidad Catolica de Chile

\section{Fanny Leyton}

University of Valparaiso School of Medicine: Universidad de Valparaiso Facultad de Medicina

Javier Moran

Valparaiso University: Universidad de Valparaiso

María José León

Pontifical Catholic University of Chile: Pontificia Universidad Catolica de Chile

\section{Catalina Sieverson}

Pontifical Catholic University of Chile: Pontificia Universidad Catolica de Chile

Antonia Muzard

Pontifical Catholic University of Chile: Pontificia Universidad Catolica de Chile

Carolina Honorato

Pontifical Catholic University of Chile: Pontificia Universidad Catolica de Chile

Karin Ensink

Laval University: Universite Laval

Norka Malberg

Yale University Child Study Center

\section{Patrick Luyten}

UCL: University College London

Stefanella Costa-Cordella ( $\nabla$ stefanella.costa@mail.udp.cl)

Millennium Institute for depression and Personality Research (MIDAP) https://orcid.org/0000-00029929-9238

\section{Study Protocol}

Keywords: Video-Feedback, Mentalization, Parental sensitivity, Postpartum Depression, Internet-based intervention. 
Posted Date: May 6th, 2021

DOl: https://doi.org/10.21203/rs.3.rs-458921/v1

License: (c) (i) This work is licensed under a Creative Commons Attribution 4.0 International License. Read Full License 


\section{Abstract}

Background: Depressive symptoms in the postpartum period are a highly prevalent mental health problem. COVID-19 restrictions have increased maternal clinical symptoms during the postpartum period, leading to a high demand for mental health assistance and the need for cost-effective care adjusted to this new context. Video feedback (VF) has been a particularly cost-effective strategy in early interventions focused on maternal sensitivity. However, these interventions usually do not include mentalization as one of the critical elements involved in sensitive and attachment relationships. Also, these interventions are generally carried out in face-to-face settings. This study's main goal is to evaluate the feasibility and acceptability of a brief internet mentalization informed with VF to improve the sensitive response in mother-baby dyads with postpartum depressive symptoms attended in primary health centers.

Methods/design: This randomized feasibility trial will include 60 mother-baby dyads. Participants will be randomized and allocated in a 1:1 ratio. A random number sequence will be computer-generated in varying block sizes (2\&4) and stratified by center. The intervention aims to improve maternal sensitivity and mentalizing by using a model based on video-feedback methodology in a virtual modality. The intervention consists of four sessions plus one pre-intervention assessment session and psychoeducational cards. Control dyads will receive only psychoeducational cards once a week. Additionally, dyads from both groups will receive the health center's treatment as usual. A MI-VF manual guides each intervention session, but its application is tailored to each dyad, VF therapists will receive one weekly group supervision session. Data will be collected at baseline, 4 weeks and 3 months followup. The main goal of this trial is to assess feasibility outcomes (recruitment rate and data completion) and change in maternal sensibility.

Discussion: This study will inform parameters for the future implementation of a large randomized controlled clinical trial, contributing to the current scarce evidence on mentalization informed interventions that use VF as their primary intervention strategy. Also, this study will provide a costeffective internet-based intervention model, feasible to be implemented in public health settings.

\section{Trial registration: NCT04748731}

\section{Background}

Maternal depressive symptoms during the perinatal period is a global mental health problem ${ }^{12}$ that has a detrimental impact on maternal sensitivity, infant's development and the mother-infant relationship ${ }^{3}$. A growing body of research recognizes the critical role of parental sensitivity and mentalizing in fostering positive parent-child relationships. This, in turn, is fundamental for a child's mental health and intergenerational transmission of attachment. Several psychosocial interventions have been developed to address maternal depressive symptoms and the mother-infant relationship's quality. Video-feedback 
interventions are a brief and cost-effective tool to foster maternal sensitivity and enhance infant's attachment security ${ }^{456-9}$.

Research on parenting and childhood development shows the importance of considering mental aspects underlying behavior to understand parent-child interactions ${ }^{10,11}$. This is why, several parenting interventions are currently focusing not only on behavioral aspects of interactions but also on the mental states they might be rooted in. This issue has been studied in terms of parental mentalizing, which plays a crucial role in fostering the adult's sensitive response and attachment security ${ }^{1213}$. Parental mentalizing is fundamental for developing affects and self-regulation ${ }^{13-19}$, the intergenerational transmission of attachment ${ }^{19,20}$ and the development of social and reflective abilities in childhood ${ }^{21}$. However, video-feedback interventions to improve parental sensitivity, often lack an explicit consideration of mentalizing as one of the key processes involved in maternal sensitivity.

Since early 2020 the COVID-19 pandemic has affected mental health worldwide, particularly in the most vulnerable groups. Recent studies show high levels of pandemic-related stress in women during the postpartum period ${ }^{2223}$ and a higher risk of mother and child mental health in this period ${ }^{24,25}$. Thus, this context demands the delivery of effective, brief and internet-based interventions, which have been shown to be effective in reducing the risk of major depressive disorder in adults with subclinical depression ${ }^{26}$ and preventing its impact on infant's development and attachment quality. The need for these interventions is clear. However, there is a scarcity of internet-based video-feedback intervention for mother-infant dyads, even though they have shown promising results 27

In response, this study aims to provide a brief online intervention based on mentalizing and using videofeedback to improve parental sensitivity in depressed mother-child dyads users of Chilean public health services. Specifically, we expect the intervention to address the following aspects: to contribute with a low-cost, evidence-based intervention suitable for being scaled-up (considering the high internet access in Chile ${ }^{28}$ ) and to provide an accessible mental health intervention during the postpartum period, especially in sanitary contexts in which face to face attention is restricted ${ }^{29}$.

\section{Aims and objectives}

This article's objective is to report on the protocol comprising a feasibility trial of online VF to improve sensitivity in mothers-baby dyads with depressive symptoms. As well as detailing the intervention, this paper provides an account of the plan to collect both quantitative and qualitative measurements of the outcome.

\section{Methods/design}

Trial design 
A small randomized controlled feasibility trial incorporating a qualitative component has been designed to assess feasibility and acceptability of a brief online intervention using VF and to collect parameters that may serve as the rationale for the implementation of a large randomized clinical trial (RCT) in the future. See Figure 1.

\section{Settings and participants}

The research will take place in five Chilean public primary health centers located in Santiago, Valparaíso and Puerto Varas. The quantitative aspects of the study will be conducted amongst mothers with depressive symptomatology and babies aged between 4 and 12 months old, enrolled at the Centers. The sample will comprise all mothers that meet the inclusion criteria, do not meet exclusion criteria and wish to participate during recruitment period (starting in March 2021 and ending in January 2012). The expected sample size is 60 mother-baby dyads in total, 30 for the control group and 30 for the experimental group. Participants will be randomized to control group or experimental group in a 1:1 ratio. A random number sequence will be computer-generated in varying block sizes (2\&4) and stratified by center. If by January 2022 the total sample has not been met, more dyads will be recruited in order to reach the sample size.

\section{Inclusion Criteria:}

Mothers must:

- Be over 18 years old

- Have a 4 to 12 month old infant

- Be a user of primary public health

- Score at least 5 points in the EPDS during routine health controls in health centers

- Speak Spanish fluently

\section{Exclusion Criteria:}

- Mothers presenting severe intellectual deficit or psychotic symptoms.

- Infants presenting severe developmental disorders (intellectual impairment and/or severe developmental delay) and/or at high risk of abuse or neglect (current domestic violence, current legal process for children rights infringement, primary caregiver in conflict with the law)

For the qualitative component of the study, a group of 4 mothers from each center will be interviewed using a semi-structured interview. Additionally, three therapists and three health professionals from the public health center will attend a focus group.

\section{Power calculation}


As a feasibility study, no hypothesis will be tested and, therefore, a formal power calculation is not required ${ }^{30,31}$. Considering similar studies and general recommendations for pilot studies ${ }^{32,33}$. We aim to recruit 60 participants in order to estimate key parameters for a future RCT.

\section{Treatment}

\section{Control group: treatment as usual (TAU) + psychoeducational cards}

The control group mother-baby dyads will receive psychoeducational cards about parenting and, just like the experimental group, they will receive the usual attention from the public health center (development monitoring, breastfeeding support, maternal depressive symptoms monitoring)

\section{Intervention arm: TAU + Video Feedback + psychoeducational cards}

The proposed intervention has been developed considering other evidence-based interventions using $\mathrm{VF}$ with mother-baby dyads like: Attachment and Biobehavioral Catch-up (ABC), (Dozier, \& Bernard, 2017), Video-feedback Intervention to promote Positive Parenting VIPP-SD ${ }^{34}$, Group Attachment-Based Intervention $(G A B I)^{35,36}$, Minding The Baby ${ }^{37,38}$, Video Intervention Therapy $(V I T)^{8}$, ODISEA mode ${ }^{39,40}$ And two Chilean interventions conducted by clinical researchers from this study, aimed at fostering parental sensitivity, improving quality in family relationships, and improving socio-emotional childhood development ${ }^{41-43}$. This Mentalization Informed VF (MI-VF) intervention is explicitly tailored to suit the needs of mothers with depression and their 4-12 months old babies. It consists of 4 sessions and is suitable to be delivered remotely. The implementation model considers 30 hours of training for therapists, including weekly supervision in groups in which videos are analyzed and each case is discussed.

The present intervention aims to improve parental sensitivity and mentalizing in depressed mothers by using a model based on video feedback methodology in a virtual modality. The whole intervention consists of four sessions plus one pre-intervention assessment session. Additionally, there is one weekly group supervision, along with the therapist independent work on the case between sessions. Each intervention session has the same structure (see figure 2).

The whole intervention process will be carried out through online methodology by trained clinical psychologists and, in addition, it will be recorded for the purpose of clinical work under supervision.

Elements of the intervention process are described below:

Pre-intervention assessment. Duration: 90 minutes (see section "measures"). The main goal of this assessment is to collect the necessary information in order to evaluate the mother (EDPS; PHQ-9, CTQ-SF, ECR), the baby (ASQ:SE-2) and the dyad (Sociodemographic information questionnaire, Video recording of free mother-baby play at the end of the session; MINI PRFI). In this session the first video recording of the mother-infant interaction is carried out. Also, an examination of the mother's concerns regarding the child, their relationship or about her as a mother is performed together with a discussion of the mother's reason for referral and intervention goals, integrating mother's concerns. 
THERAPIST INDEPENDENT WORK: 60 minutes in duration, weekly frequency. The main goal of this element is the review of the case's background information, video analysis and VF script elaboration.

The free play video -recorded in the initial assessment is watched and analyzed, and then resources and opportunities are identified. The general goal of the intervention is defined considering the mother's concerns and the reason for referral defined in the initial assessment.

Segments of 30 and 60 seconds $^{[1]}$ are selected by stopping the video at the end of the interaction sequence. Both the start and end times are written down on the script. It is recommended to show the complete video unless negative segments are identified (i.e., segments in which the mother appears as hostile or insensitive, when the child appears severely under regulated). In such cases, it might be necessary to consider only positive sequences, or selecting specific images within the video (i.e., it is not recommended to show the complete video in those cases, but only images of specific moments).

Please note: It is necessary to stop the video when both participants are seen in a positive moment of the interaction because this will be the image on which the reflection will be made.

Specific goals to be achieved in each selected segment must be identified (for example, to enhance mentalizing.)

Comments and questions to be made by the therapist should be defined, and these should be linked to the goal of each selected segment (e.g., How do you imagine that your baby felt when you took her hands and you sang her song together?)

Supervision. Duration 120 minutes, weekly frequency and group methodology.

The main goal of this element is to reflect and orient the therapeutic processes.

After the therapist prepare his/her first case, they must present to the group at least 1 complete VF process. Then, they must present parts of the other cases they will address. In the first case, the therapist must present a comprehensive diagnosis of the case integrating the initial assessment's results, the complete videos, the completed analyses, the segment selected and the elaborated script. At subsequent sessions the therapist would have to present only particular aspects of each case or about his work as therapist rather than the whole process.

The supervision has a reflective methodology ${ }^{44}$ and the experience of each one of the intervention's participants is considered (i.e., supervisor, child, caregiver and therapist). In the supervision space the supervisor also can act as a supportive figure for the therapist. This, in time, helps to make sense of the family's experiences ${ }^{35}$.

The supervision space is necessary both for the therapists training and as an opportunity for processing their own experiences and emotions related to the work with the families. In this way, the supervision promotes: a) the reflective functioning of the therapists, particularly regarding the family's interactions; $b$ ) 
the therapist as a "collaborator" of the caregivers, a role from which he/she can strengthen the caregivers' resources and abilities and those of the child, through the reflective space that video-feedback allows.

Video-feedback intervention's sessions. 60 minutes in duration, weekly. The main goal is to work with the dyad considering the therapeutic focus selected for the whole process and for each session. It is delivered online and by the same therapist that made the initial assessment.

Checking outcomes from the previous sessions. Starting from session 2. A space for discussing the caregiver's perceptions about changes in the relationship related to the tasks defined in the former session.

Video Feedback. The topic of each session addresses the contents of the video recorded in the previous session. First, the structure of the session is explained to the mother. Next, the mother is asked about the video recorded -this is useful to check if what she remembers is consistent with what was observed-. After that, the goal of the Video Feedback intervention is presented to the mother, explaining its benefits and exploring their perceptions around this issue. Then, the video segments previously selected are shown.

The selected segments are addressed as follows: 1) Observe; to attentively observe the segments checking that the mother is able to watch and to listen correctly. 2) Describe; the mother is asked to observe and describe objectively (i.e., without making inferences) what she is watching, in order to check that both caregiver and therapist are watching the same and, therefore, reflecting about the same content. 3) Mentalize; to reflect on a dialogue with the mother drawn from the comments and questions stated on the video-feedback script. The mother is encouraged to reflect on the possible mental states that motivated each member of the dyad's behaviors and its implications. She is also invited to reflect on how those mental states are present in everyday life.

It is recommended to show the complete video unless negative segments are identified in which the mother shows herself as hostile and/or insensitive or if the child is too disorganized. In those cases, the sequences with positive aspects should be considered or otherwise pictures of specific moments can be selected instead of the video.

Important: The video must be stopped when both participants are seen in a positive moment of the interaction because this will be the image they reflect on.

Video Recording. Each session has a moment in which a new interaction following a new instruction is recorded (e.g., playing, feeding, singing).

Post video interview: After each video recording, a semi-structured interview is carried out briefly addressing the mother's experience within the interaction (e.g., What was the most pleasant aspect of the interaction with your baby?), the baby's experience (e.g., What do you think was the most pleasant aspect of the interaction for your baby?), and the experience of both mother and child (e.g., Has this type of interaction with your baby happened before?) 
Task's definition: behaviors or activities to perform during the week are defined at the end of the session. These can be proposed by the therapist or emerge from what the mother learnt in the video feedback. Examples: playing 5 minutes, 3 times per week with; observe the baby's preferences in a specific context, support the baby in reaching a new ability; among others.

\section{Procedure}

Mothers with 4- to 12-month-old babies attending primary public health centers will be invited to participate by a healthcare professional. On participation agreement, they will be contacted to perform a first interview to assess eligibility (5 points on EDPS) and to sign an informed consent and a letter of authorization for their baby's participation. At any time, participants could drop the study by contacting the study coordinator (same person who make first contact with them).

Once the dyads are enrolled in one of the two groups, the pre-intervention assessment will be carried out, which evaluates: depressive symptoms (EDPS, PHQ-9), Maternal sensitivity (EAS), child's socio-emotional development (ASQ-SE), adult attachment (ECR), adverse experiences during childhood- short form (CTQSF) credibility and expectative of the treatment (CEQ) and therapeutic progress (CORE-OM). After that, interventions for both groups will be implemented followed by the post intervention assessments and, after three months of the intervention's ending, a follow-up assessment. Evaluations and interventions are detailed in figure 3.

\section{Randomization and blinding}

Participants will be randomly assigned to either $C G$ or $E G$ in a 1:1 ratio. A random number sequence will be computer-generated from permuted block sizes 4 and 6 using studyrandomizer ${ }^{\circledR}{ }^{45}$. The sequence will be administered by an external person who will be contacted by the study's coordinator each time a participant joins the study.

The outcome assessors will be blinded (even though participants and therapists will not).

\section{Outcomes}

\section{Feasibility Parameters}

The feasibility will be evaluated in terms of eligibility rates, recruitment rates and reason for study refusal, data attrition and follow-up by treatment condition.

\section{Acceptability of the intervention}

The intervention's acceptability will be assessed through in-depth interviews carried out to the mother participants. Additionally, a focus group with therapists and healthcare workers from the referral centers will be performed to explore their assessment of and levels of satisfaction with the intervention. 
The secondary outcomes will be changes in maternal sensitive response, reflective functioning, depressive symptomatology and socioemotional development, as detailed in figure 4 .

\section{Instrument description}

1. Sociodemographic information questionnaire: elaborated by the research team with the goal of describing the participant's sociodemographic, clinical and family background. It will be applied during the initial pre-intervention assessment.

2. Emotional Availability Scale EAS ${ }^{46}$. This scale will be used to codify the 15 -minute mother-infant interaction video to assess maternal sensitivity, the primary outcome of this intervention. The research team has 3 members trained and certificated in this scale. This will ensure an adequate interrater reliability. This scale and its required videos will be recorded during the pre, post and followup assessments and subsequently codified.

3. MINI Parental Development Interview, MINI-PRFI ${ }^{47}$. After the video recordings, this interview will be applied to assess reflective parental functioning or mentalizing, which is the secondary outcome of this study. This instrument uses the Slade et al., (2004) codification system. The interviews will be recorded, transcribed and codified. The research team has 4 members trained and certificated in this scale. This will ensure an adequate interrater reliability.

4. Ages and Stages Questionnaire, ASQ-SE ${ }^{48}$. Screening instrument that assesses difficulties in children's socioemotional development from 3 to 60 months. Eight questionnaires are available for different age groups: $6,12,18,24,30,36,48$, and 60 months of age. Each screens for self-regulation, compliance, communication, adaptive behaviors, autonomy, affect, and interaction with people. The Spanish version will be used, which has shown good psychometric properties. It will be used in the pre, post and follow-up assessments.

5. CORE Outcome Measure (CORE System Group ${ }^{49}$. This self-report measure comprises 18 items and assesses therapeutic outcomes focusing on the following dimensions: Well-being; Problems/symptoms: Anxiety levels, depression, trauma and physical symptoms; Functioning: interpersonal, social and general functioning in daily life; Risk/harm' includes items covering harm to self and suicidal ideation (risk-to-self items) and violent behavior and threats towards other people (risk-to-others items). Each item is scored on a Likert scale that goes from 0 to 5. CORE OM has shown high reliability (Cronbach alpha of .7 and .9 in all subscales ${ }^{50}$. It will be used in the pre, post and follow-up assessments, as well as in each intervention session.

6. Patient Health Questionnaire, PHQ-951. Depressive Symptomatology Covariable. Self-report questionnaire comprising 9 items scored in a 0-3 Likert scale developed for depression screening. It has been validated for the Chilean population ${ }^{52}$. The PHQ-9 has shown high reliability (Cronbach $=.89$ ) and an adequate convergent validity (Hamilton scale $r=.75$ ) Sensitivity analyses demonstrate that the PHQ-9 allows the identification of $86.4 \%$ of major depression cases. It will be used in the pre, post and follow-up assessments. 
7. Edinburgh Postnatal Depression Scale, EDPS ${ }^{53}$. Depressive Symptomatology Covariable. The EDPS self-report questionnaire comprising 10 items developed for depression screening. Has shown a $76.7 \%$ of sensitivity and a $92.5 \%$ of specificity for perinatal depression. Of a total of 30 points, scores of 12 or higher indicate probabilities of depression. It will be used in the pre, post and follow-up assessments

8. Experience in close relationships, ECR ${ }^{54}$. Self-report questionnaire comprising 36 items assessing adult attachment anxiety and avoidance dimensions; each dimension corresponds to a scale, with 18 items each, assessed on a 7-point Likert scale where 1 means "totally agree" and 7 means "totally disagree". The ECR has shown high reliability in its Chilean adaptation, which range from .81 and .84 in both subscales ${ }^{55}$.

9. Childhood Trauma Questionnaire- Short Form (CTQ-SF $\left.{ }^{56}\right)$. CTQ-SF is a 28- item self-report questionnaire that retrospectively assesses adverse childhood experiences in adolescents and adults. Its development was based on the 70-item instrument constructed by Bernstein et al., (1994) through an exploratory and confirmatory factorial analysis. It evaluates five types of childhood adverse experience: emotional abuse, physical abuse, sexual abuse, emotional neglect and physical neglect, with five items per scale. Each item is answered through a 5-point Likert scale that goes from almost never true to almost always true. Three additional items allow the identification of socially accepted responses or false trauma reports. Internal consistency coefficients for the English version are between 0.84 to 0.89 for emotional abuse, 0.81 to 0.89 in physical abuse, 0.92 to 0.95 in sexual abuse, 0.85 to 0.91 for emotional neglect and 0.61 to 0.78 for physical neglect ${ }^{56}$. It was validated for Chilean population, evidencing adequate psychometric properties ${ }^{57}$

10. Credibility/Expectancy Questionnaire, $\mathrm{CEQ}^{58}$. The CEQ assesses acceptability of the online intervention. It is a self-report questionnaire comprising 6 items scored in a 9-points Likert scale ranging from 1 to 9 and two factors (credibility and expectative) that explain the $82.46 \%$ of the variance. The questionnaire has shown adequate reliability with a Cronbach's Alpha of .80. The CEQ will be used in the pre, post and follow-up assessments

11. Semi-structured interview for qualitative study. During the development of the research 3 scripts for interviews will be applied, all of them at the end of the intervention. The scripts consist of:

a. First script: to explore the participant's subjective experience in depth regarding their perception of the intervention, adherence and usefulness.

b. Second script: to interview key healthcare workers from the partner health centers aiming to gather information regarding feasibility of developing this intervention. Questions are aimed at analyzing the resources needed and the impact of the intervention in the center's usual operation.

c. Third script: for interviewing the intervention's therapists to know their perspective regarding the intervention's modality, their perception on the difficulties and benefits for mothers and for them as therapists. Interviews were designed for the principal investigator together with the research team and can be found as an appendix.

Page $11 / 21$ 
[1] It has been found that the frequency and quality of the "in vivo" feedback to parents predict the size of the change after the interventions as well as the improvement in parental sensitivity (Caron, Bernard, \& Dozier, 2018).

\section{Research governance and ethics}

\section{Trial management}

The study will comply with local research governance requirements.

\section{Ethics}

Full ethical approval was obtained from the local Ethics Committee (Comité Ético Científico de Medicina, Pontificia Universidad Católica de Chile, ID 201201009, date 17-12-20). Informed and written consent will be obtained from the participants.

\section{Discussion}

The present study emerges as a response to the necessity of offering preventive evidence-based interventions for mother-baby dyads that are part of the risk population in sanitary contexts in which face to face attention is restricted. In this scenario, an internet-based attention offers an optimal modality that has shown to be effective in the treatment of several mental health problems. This is especially relevant in the current context of COVID-19 pandemic.

This modality could become relevant considering that spontaneous mental health consultations related with postpartum depression are scarce ${ }^{59}$, and its prevalence is high ${ }^{2}$.

It is known that women with postpartum depressive symptoms are less likely to seek mental health attention in part because of the overloads of baby's care and also because of stigma ${ }^{606162}$.

Internet based interventions could be helpful in facing these difficulties ${ }^{63}$. Emerging research shows that e-mental health can be a very useful tool for addressing mental health problems in infancy ${ }^{6465}$. We expect this research to contribute with evidence in this area. Moreover, this study offers a novel approach to parenting interventions using reflective functioning in the context of video feedback.

The present research aims to demonstrate the feasibility of implementing an online MI-VF intervention to improve maternal sensitivity, one of the strongest predictors of children attachment quality. It also intends to improve other maternal mental health aspects and baby's socioemotional development to have an additional estimation of the intervention's effect. The results of this study might allow the acquisition of implementation parameters that can be useful for a future effectiveness trial to assess the impact of the intervention in depressive symptomatology during the postpartum period.

\section{Declarations}


Full ethical approval was obtained from the local Ethics Committee (Comité Ético Científico de Medicina, Pontificia Universidad Católica de Chile, ID 201201009, date 17-12-20). The study will be conducted in accordance with the ethical principles that have their origin in the Declaration of Helsinki (1996) and the principles of Good Clinical Practice (such as data storage and administrative functions).

Written informed consent will be obtained from participants in the study (adults and children). Participants will be free to withdraw from the study at any time without giving a reason and without their care being affected. All the information collected during this trial will be confidential

Interviews which are transcribed will be anonymized at the point of transcription. Any third party involved with transcribing of interviews will sign a confidentiality agreement and be fully instructed in how to anonymize transcripts. There is no financial compensation for participating in the study.

\section{Consent for publication}

Not applicable.

\section{Availability of supporting data}

The datasets used during the current study will be available from the corresponding author on a reasonable request.

\section{Competing interests}

The authors declare that they have no competing interests.

\section{Funding}

This work was funded by the ANID Millennium Science Initiative / Millennium Institute for Research on Depression and Personality-MIDAP ICS13_005

\section{Authors' contributions}

$\mathrm{MO}, \mathrm{FL}, \mathrm{SC}, \mathrm{JM}, \mathrm{ML}, \mathrm{CS}, \mathrm{AM}, \mathrm{CH}$ authors conceived the study and are involved in managing the project. $\mathrm{KE}, \mathrm{NB}, \mathrm{PL}$ advised on the construction of the intervention manual for this study. All authors contributed to the drafting of this paper and approved the final manuscript.

\section{Acknowledgements}

We would like to express our gratitude for the support and funding from the ANID Millennium Science Initiative /Millennium Institute for Research on Depression and Personality-MIDAP ICS13_005

\section{Authors' information}


MO is the principal investigator of this study. She is Associated Researcher of the Millennium Institute for Research on Depression and Personality-MIDAP ICS13_005 and Associated Professor, School of Psychology, Pontificia Universidad Católica de Chile.

\section{Trial registration}

NCT04748731. Registered on February 10, 2021 https://clinicaltrials.gov/ct2/show/NCT04748731.

\section{Trial status}

The study started recruiting patients in March 2021. Recruitment will end by January 2022.

\section{References}

1. Jadresic E, Depresión en el embarazo y el puerperio. Revista chilena de neuro-psiquiatría 48, 269278 (2010).

2. Hahn-Holbrook J, Cornwell-Hinrichs T, Anaya I. Economic and Health Predictors of National Postpartum Depression Prevalence: A Systematic Review, Meta-analysis, and Meta-Regression of 291 Studies from 56 Countries. Front Psychiatry. 2017;8:248.

3. Slomian $\mathrm{J}$, Honvo G, Emonts $\mathrm{P}$, Reginster $\mathrm{J}-\mathrm{Y}$, Bruyère $\mathrm{O}$. Consequences of maternal postpartum depression: A systematic review of maternal and infant outcomes. Womens Health. 2019;15:1745506519844044.

4. Bakermans-Kranenburg MJ, van IJzendoorn MH, Juffer F. Less is more: meta-analyses of sensitivity and attachment interventions in early childhood. Psychol Bull. 2003;129:195-215.

5. Fukkink RG. Video feedback in widescreen: a meta-analysis of family programs. Clin Psychol Rev. 2008;28:904-16.

6. Steele $M$, et al. Looking from the outside in: the use of video in attachment-based interventions. Attach Hum Dev. 2014;16:402-15.

7. Høivik MS, et al. Video feedback compared to treatment as usual in families with parent-child interactions problems: a randomized controlled trial. Child Adolesc Psychiatry Ment Health. 2015;9:3.

8. Facchini S, Martin V, Downing G, Pediatricians W-B. Visits, and Video Intervention Therapy: Feasibility of a Video-Feedback Infant Mental Health Support Intervention in a Pediatric Primary Health Care Setting. Front Psychol. 2016;7:179.

9. O'Hara L, et al. Video feedback for parental sensitivity and attachment security in children under five years. Cochrane Database Syst Rev. 2019;11:CD012348.

10. Fonagy P, Gergely G, Jurist EL. Affect Regulation, Mentalization and the Development of the Self. Routledge; 2018.

11. Sharp C, Fonagy P. The parent's capacity to treat the child as a psychological agent: Constructs, measures and implications for developmental psychopathology. Soc Dev. 2008;17:737-54. 
12. Borelli JL, et al. Maternal and Child Sexual Abuse History: An Intergenerational Exploration of Children's Adjustment and Maternal Trauma-Reflective Functioning. Front Psychol. 2019;10:1062.

13. Zeegers MAJ, Colonnesi C, Stams G-JJM, Meins E. Mind matters: A meta-analysis on parental mentalization and sensitivity as predictors of infant-parent attachment. Psychol Bull. 2017;143:1245-72.

14. Ensink K, Normandin L, Plamondon A, Berthelot N, Fonagy P. Intergenerational pathways from reflective functioning to infant attachment through parenting. Can. J. Behav. Sci. 48, 9-18.

15. Handbook of mentalizing in mental health practice. 593. (2012).

16. Meins E, Fernyhough C, Fradley E, Tuckey M. Rethinking maternal sensitivity: mothers' comments on infants' mental processes predict security of attachment at 12 months. J Child Psychol Psychiatry. 2001;42:637-48.

17. Oppenheim D, Koren-Karie N. Mothers' insightfulness regarding their children's internal worlds: The capacity underlying secure child-mother relationships. Infant Mental Health Journal. 2002;23:593605.

18. Slade A. Parental reflective functioning: an introduction. Attach Hum Dev. 2005;7:269-81.

19. Slade A, Grienenberger J, Bernbach E, Levy D, Locker A. Maternal reflective functioning, attachment, and the transmission gap: a preliminary study. Attach Hum Dev. 2005;7:283-98.

20. Fonagy P, Steele H, Steele M. Maternal representations of attachment during pregnancy predict the organization of infant-mother attachment at one year of age. Child Dev. 1991;62:891-905.

21. Borelli JL, et al. School-Aged Children With Higher Reflective Functioning Exhibit Lower Cardiovascular Reactivity. Front Med. 2018;5:196.

22. Committee I-AS, Others. Addressing mental health and psychosocial aspects of COVID-19 outbreak. See https://interagencystandingcommittee. org/system/files/2020-03/IASC\% 20Interim\% 20Briefing\% 20Note\% 20on\% 20COVID-19\% 200utbreak\% 20Readiness\% 20and\% 20Response\% 200perations (2020).

23. Olhaberry M, Sieverson C. \& P., F. The impact of COVID-19 on experiences of pregnancy and/or parenting in Chile. Journal of Infant Mental Health (2021).

24. Wang G, Zhang Y, Zhao J, Zhang J, Jiang F. Mitigate the effects of home confinement on children during the COVID-19 outbreak. Lancet. 2020;395:945-7.

25. Jiao WY, et al. Behavioral and Emotional Disorders in Children during the COVID-19 Epidemic. J Pediatr. 2020;221:264-6.e1.

26. Buntrock C, et al. Preventing Depression in Adults With Subthreshold Depression: Health-Economic Evaluation Alongside a Pragmatic Randomized Controlled Trial of a Web-Based Intervention. J Med Internet Res. 2017;19:e5.

27. Baker M, Biringen Z, Meyer-Parsons B, Schneider A. Emotional attachment and emotional availability tele-intervention for adoptive families. Infant Ment Health J. 2015;36:179-92. 
28. Pinto M, González B. Séptima encuesta de acceso, usos y usuarios de internet. Santiago: Subsecretaría de telecomunicaciones (2016).

29. Rojas G, Martínez V, Martínez P, Franco P. \& Jiménez-Molina, Á. Improving Mental Health Care in Developing Countries Through Digital Technologies: A Mini Narrative Review of the Chilean Case. Front Public Health. 2019;7:391.

30. Leon AC, Davis LL, Kraemer HC. The role and interpretation of pilot studies in clinical research. J Psychiatr Res. 2011;45:626-9.

31. Eldridge SM, et al. CONSORT 2010 statement: extension to randomised pilot and feasibility trials. BMJ. 2016;355:i5239.

32. Kalinauskiene $L$, et al. Supporting insensitive mothers: the Vilnius randomized control trial of videofeedback intervention to promote maternal sensitivity and infant attachment security. Child Care Health Dev. 2009;35:613-23.

33. Sim J, Lewis M. The size of a pilot study for a clinical trial should be calculated in relation to considerations of precision and efficiency. J Clin Epidemiol. 2012;65:301-8.

34. van ljzendoorn FJMJB-KMH. Promoting Positive Parenting:An Attachment-Based Intervention. Taylor \& Francis; 2007.

35. Murphy A, et al. Group Attachment-Based Intervention. Fam Community Health. 2015;38:268-79.

36. Steele H, Murphy A, Bonuck K, Meissner P, Steele M. Randomized control trial report on the effectiveness of Group Attachment-Based Intervention (GABIC): Improvements in the parent-child relationship not seen in the control group. Dev Psychopathol. 2019;31:203-17.

37. Slade A, et al. Minding the Baby®: Enhancing parental reflective functioning and infant attachment in an attachment-based, interdisciplinary home visiting program. Dev Psychopathol. 2020;32:12337.

38. Sadler LS, Slade A, Mayes LC. Minding the baby: A mentalization-based parenting program. Handbook of mentalization-based treatment 271-288 (2006).

39. Gómez E, Maureira M Modelo de video-feedback ODISEA 2.0. Oportunidades para el desarrollo de interacciones sensibles, eficaces y afectivas. (2017).

40. Gómez E, Muñoz M Manual para el modelo de video-feedback. oportunidades para el desarrollo de interacciones sensibles, eficaces y afectivas. Material no publicado. (2013).

41. Olhaberry $M$, et al. Is it possible to improve early childhood development with a video-feedback intervention directed at the mother-father-child triad? Res Psychother. 2019;22:324.

42. Olhaberry $M$, et al. Video-feedback intervention to improve parental sensitivity and the quality of interactions in mother-father-infant triads. Interactions. 2017;27:0-000.

43. Olhaberry $M$, Leòn MJ, Seguel M, Mena $C$. Video-feedback intervention in mother-baby dyads with depressive symptomatology and relationship difficulties. Research in Psychotherapy:

Psychopathology Process Outcome. 2015. doi:10.4081/ripppo.2015.177. 
44. Shahmoon-Shanok R. Reflective supervision for an integrated model: What, why, and how. Mental health in early intervention 343-381 (2006).

45. Study Randomizer. Home - Study Randomizer. https://www.studyrandomizer.com.

46. Biringen Z, Derscheid D, Vliegen N, Closson L, Ann Easterbrooks M. Emotional availability (EA): Theoretical background, empirical research using the EA Scales, and clinical applications. Dev Rev. 2014;34:114-67.

47. Ensink K, et al. Costs of Not Getting to Know You: Lower Levels of Parental Reflective Functioning Confer Risk for Maternal Insensitivity and Insecure Infant Attachment. Infancy. 2019;24:210-27.

48. Squires J, Bricker D, Twombly E The ASQ:SE user's guide: For the Ages \& Stages Questionnaires: Social-emotional. (2002).

49. Barkham M, Evans C, Margison F, McGrath G. The rationale for developing and implementing core outcome batteries for routine use in service settings and psychotherapy outcome research. Abingdon. 1998;7:35-47.

50. Trujillo A, et al. Psychometric properties of the Spanish version of the Clinical Outcomes in Routine Evaluation - Outcome Measure. Neuropsychiatr Dis Treat. 2016;12:1457-66.

51. Diez-Quevedo C, Rangil T, Sanchez-Planell L, Kroenke K, Spitzer RL. Validation and utility of the patient health questionnaire in diagnosing mental disorders in 1003 general hospital Spanish inpatients. Psychosom Med. 2001;63:679-86.

52. Saldivia S, et al. Propiedades psicométricas del PHQ-9 (Patient Health Questionnaire) en centros de atención primaria de Chile. Revista médica de Chile. 2019;147:53-60.

53. Cox JL, Holden JM, Sagovsky R. Detection of postnatal depression. Development of the 10-item Edinburgh Postnatal Depression Scale. Br J Psychiatry. 1987;150:782-6.

54. Alonso-Arbiol I, Balluerka N, Shaver PR. A Spanish version of the Experiences in Close Relationships (ECR) adult attachment questionnaire. Pers Relatsh. 2007;14:45-63.

55. Spencer R, Guzmán M, Fresno A, Ramos N. Validación chilena del cuestionario de evaluación del apego romántico Experiences in Close Relationships (ECR): análisis de la validez de criterio. Terapia. 2013;31:313-24.

56. Bernstein DP, et al. Development and validation of a brief screening version of the Childhood Trauma Questionnaire. Child Abuse Negl. 2003;27:169-90.

57. Behn A, et al. Validación de la versión en español del Childhood Trauma Questionnaire-Short Form en Chile, en una muestra de pacientes con depresión clínica. Revista médica de Chile. 2020;148:33643.

58. Devilly GJ, Borkovec TD. Psychometric properties of the credibility/expectancy questionnaire. J Behav Ther Exp Psychiatry. 2000;31:73-86.

59. Fonseca A, Gorayeb R, Canavarro MC. Women's use of online resources and acceptance of e-mental health tools during the perinatal period. Int J Med Informatics. 2016;94:228-36. 
60. O'Mahen HA, Flynn HA. Preferences and perceived barriers to treatment for depression during the perinatal period. J Womens Health. 2008;17:1301-9.

61. Kim JJ, et al. Barriers to mental health treatment among obstetric patients at risk for depression. Am J Obstet Gynecol. 2010;202:312.e1-5.

62. Rojas G, et al. Barreras de acceso a tratamiento de la depresión posparto en Centros de Atención Primaria de la Región Metropolitana: un estudio cualitativo. Revista médica de Chile. 2015;143:42432.

63. Kingston D, Rocha R. Telehealth and Women's Perinatal Mental Health. Women's Mental Health 33547 (2020) doi:10.1007/978-3-030-29081-8_23.

64. Danaher BG, et al. MomMoodBooster web-based intervention for postpartum depression: feasibility trial results. J Med Internet Res. 2013;15:e242.

65. O'Mahen HA, et al. Internet-based behavioral activation-treatment for postnatal depression (Netmums): a randomized controlled trial. J Affect Disord. 2013;150:814-22.

\section{Appendix}

The appendix is not available with this version

\section{Figures}




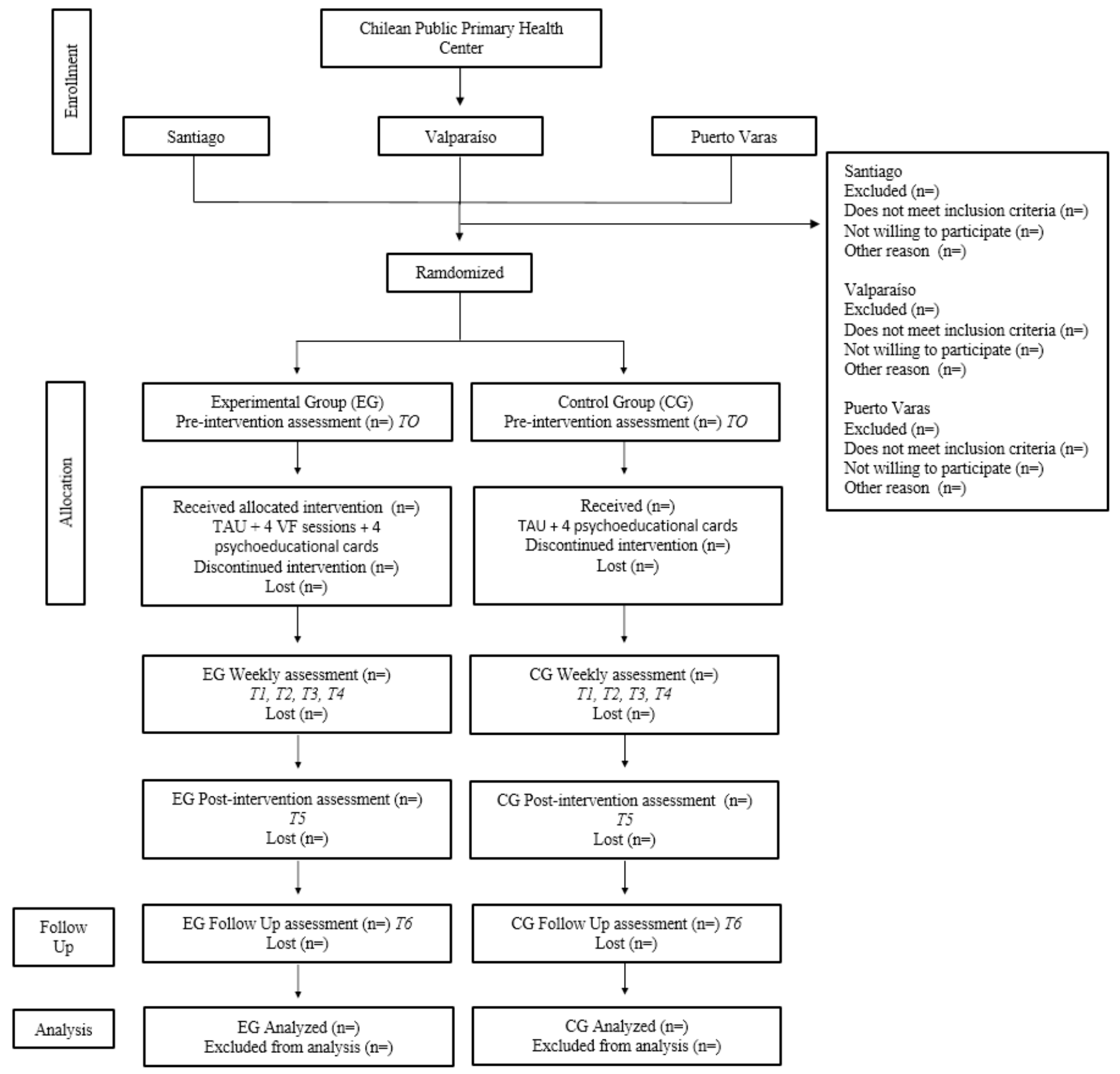

\section{Figure 1}

Flowchart of study phases and instrument application. 


\section{Pre-Intervention Assesment}

Questionnaires and Interview

Video recording

Goals discussion

Therapist's independent work

Video Analysis

Segments selection

Comments/questions definition

Each session has this 5 elements:
Checking outcomes
from the previous session

\section{Intervention's Sessions}

4 sessions

Each session has the same

elements and structure

\section{Supervision}

Online

Weekly

Reflective

\section{Figure 2}

Summary of Intervention's elements.

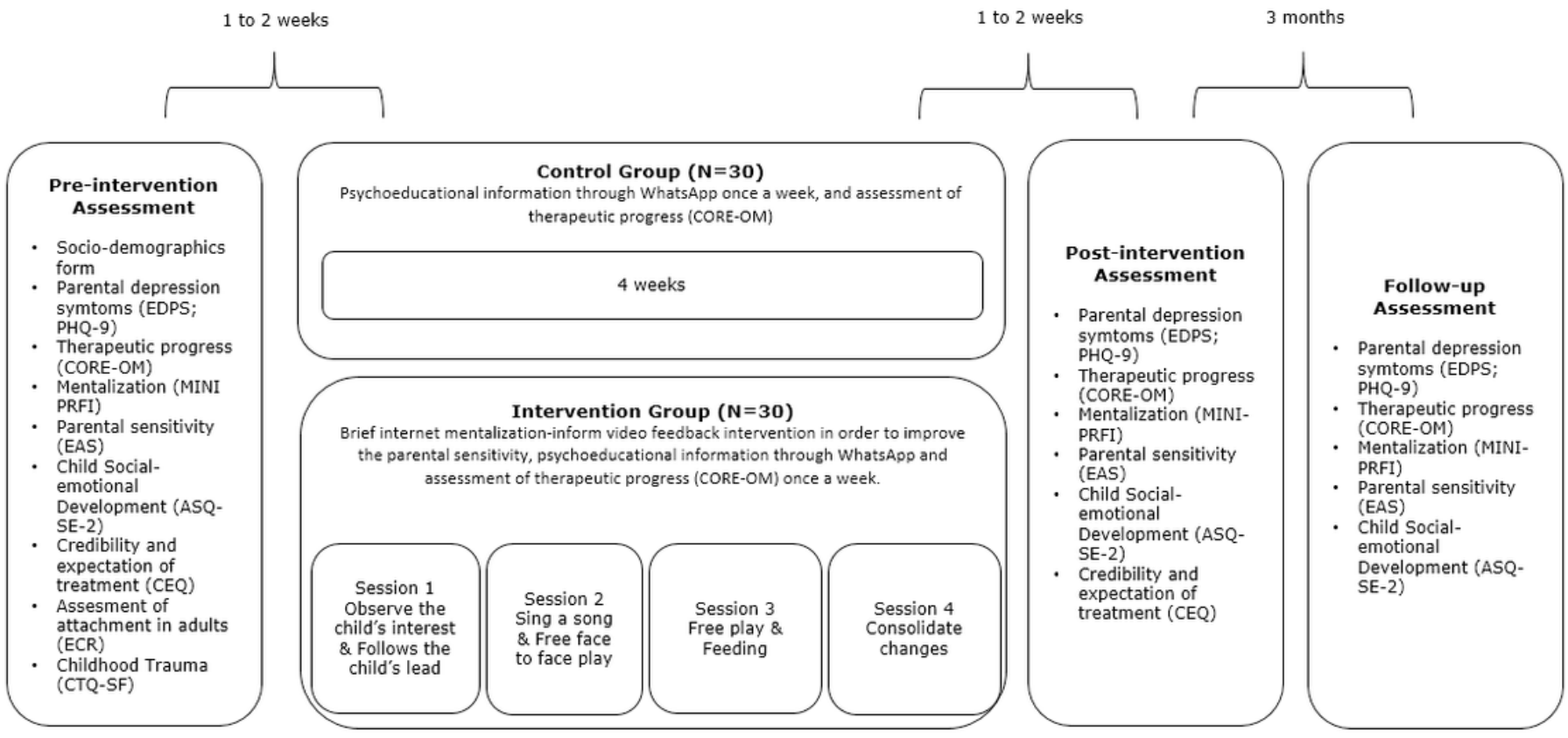

\section{Figure 3}

Assessments interventions and time intervals for control group and experimental group. 


\begin{tabular}{|c|c|c|c|c|c|c|c|c|c|c|}
\hline \multirow[b]{2}{*}{ Timepoint } & \multirow{2}{*}{\begin{tabular}{|c|} 
Enrollment \\
$\mathrm{T} 1$ \\
\end{tabular}} & \multirow{2}{*}{$\begin{array}{c}\text { Baseline } \\
\text { T0 }\end{array}$} & \multirow[t]{2}{*}{ Allocation } & \multicolumn{6}{|c|}{ Post-allocation } & \multirow[t]{2}{*}{ Close-out } \\
\hline & & & & T1 & $\mathrm{T} 2$ & T3 & T4 & T5 & T6 & \\
\hline \multicolumn{11}{|l|}{\begin{tabular}{|l|} 
ENROLLMENT \\
\end{tabular}} \\
\hline Eligibility screen & $\mathrm{x}$ & & & & & & & & & \\
\hline Informed consent & $\mathrm{x}$ & & & & & & & & & \\
\hline Allocation & & & $\mathrm{x}$ & & & & & & & \\
\hline \multicolumn{11}{|l|}{ INTERVENTION } \\
\hline VF + TAU+ psychoeducational cards & & & & $\mathrm{x}$ & $\mathrm{x}$ & $\mathrm{x}$ & $\mathrm{x}$ & & & \\
\hline TAU + psychoeducational cards & & & & $\mathrm{x}$ & $\mathrm{x}$ & $x$ & $\mathrm{x}$ & & & \\
\hline \multicolumn{11}{|l|}{ ASSESSMENT } \\
\hline \multicolumn{11}{|l|}{ Quantitative } \\
\hline EDPS & & $\mathrm{x}$ & & & & & & $\mathrm{x}$ & $x$ & \\
\hline \begin{tabular}{|l|} 
PHQ-9 \\
\end{tabular} & & $\mathrm{x}$ & & & & & & $\mathrm{x}$ & $\mathrm{x}$ & \\
\hline CORE-OM & & $\mathrm{x}$ & & $\mathrm{x}$ & $\mathrm{x}$ & $\mathrm{x}$ & $\mathrm{x}$ & $x$ & $\mathrm{x}$ & \\
\hline MINI PRFI & & $\mathrm{x}$ & & & & & & $x$ & $\mathrm{x}$ & \\
\hline EAS & & $\mathrm{x}$ & & & & & & $\mathrm{x}$ & $\mathrm{x}$ & \\
\hline ASQ-SE-2 & & $\mathrm{x}$ & & & & & & $\mathrm{x}$ & $x$ & \\
\hline CEQ & & $\mathrm{x}$ & & & & & & $\mathrm{x}$ & $\mathrm{x}$ & \\
\hline CTQ-SF & & $\mathrm{x}$ & & & & & & $x$ & $x$ & \\
\hline ECR & & $\mathrm{x}$ & & & & & & $\mathrm{x}$ & $x$ & \\
\hline Socialdemographic questionnarie & & $\mathrm{x}$ & & & & & & $\mathrm{x}$ & $\mathrm{x}$ & \\
\hline Qualitative data from caregivers & & & & & & & & & $x$ & \\
\hline Qualitative data from stakeholders & & & & & & & & & & $\mathrm{x}$ \\
\hline
\end{tabular}

\section{Figure 4}

Schedule of enrollment, interventions and assessments VF, video-feedback intervention, TAU, Treatment as usual, EDPS, Edinburgh Postnatal Depression Scale; PHQ-9, Patient Health Questionnaire; CTQ-SF, Childhood Trauma Questionnaire Short Form, CORE-OM, CORE System Group Outcome Measure; MINI PRFI, MINI Parental Development Interview; EAS Emotional Availability Scale; ASQ-SE, Ages and Stages Questionnaire; CEQ, Credibility/Expectancy Questionnaire; ECR, Experience in close relationships.

\section{Supplementary Files}

This is a list of supplementary files associated with this preprint. Click to download.

- SPIRITFillablechecklist15Aug20131.doc 